\title{
Alcuni risultati di analisi spettrale per l'operatore di Laplace su insiemi non limitati $\left(^{*}\right)(* *)$.
}

\author{
Giaxfranco Bottaro
}

Summary. - In this work I study the spectrum and a system of generalized eigenfunctions for Laplace's operator in unbounded domains: the plane, the half plane, the plane layer, the exterior of a sphere.

\section{Introduzione.}

Questo lavoro è dedicato allo studio dello spettro dell'operatore di Laplace in regioni non limitate, quali lo spazio, il semispazio, lo strato e l'esterno di una sfera.

Nel n. 1 si dà la definizione di integrale diretto di spazi hilbertiani [11] e l'enunciato di un teorema di Von NeUmann [10].

Nel n. 2 si descrive un'idea di Gärding, in [6], che ci permette: nel n. 3 di riottenere alcuni risultati di natura hilbertiana, relativi alla diagonalizzazione dell'operatore di Laplace in tutto lo spazio, conseguiti da TALEXTI in [17], nei n. 4 e 5 di rispondere alle analoghe questioni per lo stesso operatore in un semispazio, in uno strato e all'esterno di una sfera.

\section{1. - Integrale diretto e teorema di Von Neumann.}

DeFINIZIONE. - Sia:

1) $\Lambda$ uno spazio topologico localmente compatto, separabile, su cui è definita una misura $\mu$ positiva, regolare di Borel

2) $\nu: \Lambda \rightarrow\{1,2, \ldots, \infty\}$ una funzione misurabile Borel.

3) $H(\lambda)=\left\{u(\lambda) \in C^{p(\lambda)}: \sum_{j=1}^{v(\lambda)}\left|u_{j}(\lambda)\right|^{2}<\infty\right\}$ per quasi ogni $\lambda$ (rispetto a $\left.\mu\right) . H(\lambda) \grave{\mathrm{e}}$ reso spazio di Hilbert (separabile) dal prodotto sealare $(u(\lambda), v(\lambda))_{H(\lambda)}=$ $=\sum_{j=1}^{v(\lambda)} u_{j}(\lambda) \overline{v_{j}(\lambda)}$

(*) Entrata in Redazione il 29 novembre 1973.

(**) Lavoro eseguito nell'ambito del "Centro di Matematica e Fisica teorica " del C.N.R. presso l'Università degli Studi di Genova.

14 - Annali di Matematica 
Consideriamo in $\underset{\lambda \in A}{X} H(\lambda)$ il sottinsieme $H_{1}=\left\{u \in \underset{\lambda \in A}{X} H(\lambda)\right.$ tali che $u_{j}(\lambda)$ sia $\mu$-misurabile per ogni indice $j$ e $\left.\int_{A}\|u(\lambda)\|^{2} d \mu(\lambda)<\infty\right\}$.

Introduciamo in $H_{1}$ una relazione di equivalenza $\sim$, ponendo $u \sim v$ se $\int_{A} \| u(\lambda)-$ $-v(\lambda) \|^{2} d \mu(\lambda)=0$ e dotiamo lo spazio quoziente $\hat{H}=H_{1} / \sim$ di una struttura hilbertiana mediante il prodotto scalare

$$
(u, v)_{\hat{H}}=\int_{\lambda}(u(\lambda), v(\lambda))_{H(\lambda)} d \mu(\lambda)
$$

Si prova, generalizzando il teorema di Fisher Riesz, che $\hat{H}$ diventa in tal modo spazio completo ed è facile controllare che esso è altresì separabile. Indicheremo tale spazio anche con le notazioni $\int_{\Lambda} H(\lambda) d \mu$ e $L^{2}(A, \mu, v)$ e lo chiameremo spazio spettrale.

Teorema (cfr. [4]). - Sia $A$ un operatore normale limitato su uno spazio di Hilbert separabile. Allora esiste uno spazio spettrale $\hat{H}$ ed una applicazione unitaria $U: H \rightarrow \hat{H}$ tale che, indicata con $f(A)$ l'immagine di $f$ nell' isomorfismo di GELFAND Namark [12 e 10] fra le classi di equivalenza di funzioni di Baire sullo spettro di $A$ (ove $f \sim g$ significa $f=g$ quasi ovunque) e la corrispondente $C^{*}$ algebra commuta . tiva di operatori generata da $A$, si abbia:

$$
\left(U f(A) U^{*} v\right)(\lambda)=f(\lambda) v(\lambda) \quad \text { per ogni } v \in \hat{H}
$$

ed $f \in \mathfrak{B}(\sigma(A))$. Nel seguito chiameremo $U$ diagonalizzazione canonica di $A$. Con l'uso della trasformata di Cayley si può sostituire l'ipotesi $A$ normale limitato con l'ipotesi $A$ autoaggiunto.

Von Neumans ha dimostrato in questo teorema l'esistenza dell'operatore $U$; scopo di questa nota è invece costruirlo in alcuni casi particolari.

\section{2. - Un'idea di Garding per ottenere la diagonalizzazione di un operatore differenziale a coefficienti costanti.}

$\mathrm{E}$ ben noto che dato l'operatore autoaggiunto $D_{k}=\partial / i \partial x_{k}$ con dominio nelle $f \in L^{2}\left(\boldsymbol{R}^{n}\right)$ tali che $D_{k} f \in L^{2}\left(\boldsymbol{R}^{n}\right){ }^{(1)}$ se $U: H=L^{2}\left(\boldsymbol{R}^{n},||\right) \rightarrow K=L^{2}\left(\boldsymbol{R}^{n},|| /(2 \pi)^{n}\right)$ (ove | indica l'usuale misura di Lebesgue) è la trasformata di Fourier, $U$ è unitario e vale: $\left(U D_{k} U^{*}\right) v(\lambda)=\lambda_{k} v(\lambda)$, per ogni $v \in K$, tale che $U^{*} v \in \operatorname{dom} D_{k}$.

Più in generale, se $p\left(\lambda_{1}, \ldots, \lambda_{n}\right)$ è un polinomio reale (che nei successivi n. $3,4,5$ sarà $\left.p(\lambda)=\sum_{i=1}^{n} \lambda_{i}^{2}\right)$, definiamo l'operatore $B=p\left(D_{1}, \ldots, D_{n}\right)$, che è autoaggiunto se il

(1) $D_{k} f$ è intesa nel senso delle distribuzioni. 
suo dominio è ristretto alle $u$ tali che

$$
u \in L^{2}\left(\boldsymbol{R}^{n}\right) \quad \int|(U u)(\lambda)|^{2} p^{2}(\lambda) \frac{d \lambda}{(2 \pi)^{n}}<\infty\left(^{1}\right) .
$$

Potremo dire che $U$ diagonalizza $B$ in modo non canonico, in quanto risulta

$$
\left(U B U^{*} v\right)(\lambda)=p(\lambda) v(\lambda)
$$

Se ci limitiamo a considerare il caso in cui $p\left(\lambda_{i}\right)=\sum_{i j} a_{i j} \lambda_{i} \lambda_{j}$ ove $a_{i j}=a_{j i}$ e $p$ è definito positivo, si può ottenere la diagonalizzazione canonica nel modo seguente: per ogni $t \in \boldsymbol{R}$ sia $S_{t}$ la superficie (ellissoide eventualmente degenere) di equazione $p(\lambda)=t, \lambda \in \boldsymbol{R}$, e sia $N=\boldsymbol{R}_{+}$. Definiamo su $S_{i}$ la misura $n-1$ dimensionale $\omega_{i}$ in modo che risulti $d \lambda=(2 \pi)^{n} d \omega_{t} d t$.

Nello spazio di Hilbert $H_{t}=L^{2}\left(S_{t}, \omega_{t}\right)$ sia $\left\{h_{j}(t, \lambda)\right\}_{j \in N}$ un sistema o.n.c.. Consideriamo $L=\int_{N} H_{t} d t\left(\right.$ n. 1) e definiamo $V: L^{2}\left(\boldsymbol{R}^{n},|| /(2 \pi)^{n}\right) \rightarrow L$ mediante l'uguaglianza $(V v)_{i}(t)=\int_{s_{t}} v(\lambda) \overline{h_{j}(t, \lambda)} d \omega_{t}$, ove $v \in C_{0}^{\infty}\left(\boldsymbol{R}^{n}\right)$ e $j \in N$ e prolungato per continuità su $L^{2}\left(\boldsymbol{R}^{n}\right)$, (di fatto è isometrico come proveremo ora di seguito). Indicato allora con $V^{*}$ l'operatore aggiunto di $V$, per ogni $w \in L$ risulta:

$$
\left(V^{*} w\right)(\lambda)=\sum_{j=1}^{\infty} h_{j}(p(\lambda), \lambda) v_{j}(p(\lambda))
$$

la serie convergendo ovviamente in $K\left({ }^{2}\right)$. Infatti:

$$
\begin{aligned}
\left(v, V^{*} w\right)_{K}=(V v, w)_{L} & =\int_{N} \sum_{j=1}^{\infty}(V v)_{j}(t) \overline{w_{j}(t)} d t= \\
& =\int v(\lambda) \overline{\sum_{j=1}^{\infty} h_{j}(p(\lambda), \lambda) w_{j}(p(\lambda))} \frac{d \lambda}{(2 \pi)^{n}}=\left(v, \sum_{j=1}^{\infty} h_{j}(p(\lambda), \lambda) w_{j}(p(\lambda))_{K}\right) .
\end{aligned}
$$

Proviamo adesso che $V$ è unitario facendo vedere che è isometrico e surgettivo.

Sia $v \in C_{0}^{\infty}\left(\boldsymbol{R}^{n}\right)$, allora

$$
\begin{aligned}
\|V v\|_{K}^{2}=\int_{N} \sum_{j=1}^{\infty}\left|\left(v(\lambda), h_{j}(t, \lambda)\right)\right|^{2} d t=\int_{N}\|v(\lambda)\|_{L^{2}\left(S_{t}, \omega_{t}\right)}^{2} d t= \\
\quad=\int_{N} \int_{S_{t}}|v(\lambda)|^{2} d \omega_{t} d t=\int|v(\lambda)|^{2} \frac{d \lambda}{(2 \pi)^{n}}=\|v\|_{K}^{2},
\end{aligned}
$$

(1) $\int f(\lambda) d \lambda$ significa $\int_{\boldsymbol{R}^{n}} f(\lambda) d \lambda$, essendo $d \lambda$ la misura di Lebesgue su $\boldsymbol{R}^{n}$.

$\left({ }^{2}\right)$ Con $v_{j}(t)$ si sono indicati i coeffieienti di Fourier di $w(t)$ rispetto al sistema o.n.c. 
$V$ è isometrico. Verifichiamo che $V$ è surgettivo, facendo vedere che $V V^{*}=i d$. Risulta infatti

$$
\begin{aligned}
& V V^{*} w_{k}(t)=\int_{S_{t}} \sum_{j=1}^{\infty} h_{j}(p(\lambda), \lambda) w_{j}(p(\lambda)) \overline{h_{k}(p(\lambda), \lambda)} d \omega_{t}= \\
&=\sum_{j=1}^{\infty} w_{j}(t) \int_{S_{t}} h_{j}(t, \lambda) \overline{h_{k}(t, \lambda)} d \omega_{t}=w_{k}(t), \quad \text { per ogni } w \in L .
\end{aligned}
$$

Allora $W=V U: H \rightarrow L$ ̀े unitaria e diagonalizza $B$ canonicamente. Infatti: se $u \in \operatorname{dom} B$

$$
\begin{aligned}
(W B u)_{j}(t)=\left(V U p\left(D_{1}, \ldots, D_{n}\right) u\right)_{i}(t) & =\int_{s_{t}} p\left(\lambda_{1}, \ldots, \lambda_{n}\right)(U u)(\lambda) \overline{h_{j}(t, \lambda)} d \omega_{j}(\lambda)= \\
& =\int_{S_{t}} t(U u)(\lambda) \overline{h_{j}(t, \lambda)} d \omega_{j}(\lambda)=t(V U u)(t)=t(W u)(t) .
\end{aligned}
$$

Inoltre se $u \in C_{0}^{\infty}\left(\boldsymbol{R}^{n}\right)$,

$$
(W u)_{j}(t)=\int_{S_{t}} \int_{\operatorname{loxp}}[-i(x, \lambda)] u(x) d x \overline{h_{j}(t, \lambda)} d \omega_{t}(\lambda)
$$

Allora $(W u)_{j}(t)$ si può scrivere $(W u)_{j}(t)=\left\langle u, \varphi_{j}(t)\right\rangle$ con $\varphi_{j}(t)$ distribuzione. Tale distribuzione è un'antosoluzione generalizzata [1] per $B$, e cioè verifica $\left\langle B u, \varphi_{j}\right\rangle=$ $=t\left\langle u, \varphi_{j}\right\rangle$ per ogni $u \in O_{0}^{\infty}\left(\boldsymbol{R}^{n}\right)$. Risulta infatti :

$$
\left\langle B u, \varphi_{j}\right\rangle=(W B u)_{j}=\lambda(W u)_{j}=\lambda\left\langle u, \varphi_{j}\right\rangle .
$$

Le distribuzioni $\varphi_{j}(t)$ si riducono a funzioni di classe $C^{\infty}\left(\boldsymbol{R}^{n}\right)$, anche se non appartenenti a $L^{2}$ (perchè se $B$ è ellittico $S_{t}$ è compatto).

Risulta infatti

$$
\varphi_{j}(t, x)=\int_{S_{t}} \exp [i(x, \lambda)] h_{j}(t, \lambda) d \omega_{t}
$$

\section{3. - Diagonalizzazione dell'operatore di Laplace.}

Considero in $\boldsymbol{R}^{n}$ l'operatore:

1) $\Delta=-\sum_{j=1}^{n} \partial^{2} /\left(\partial x_{j}\right)^{2}=\sum_{j=1}^{n}\left(\partial / i \partial x_{j}\right)^{2}$ il cui polinomio caratteristico è $p\left(\lambda_{1}, \ldots, \lambda_{n}\right)=$ $=\sum_{j=1}^{n} \lambda_{j}^{2}=|\lambda|^{2}$. Come si è detto nel n. 3 la condizione perchè

2) $\Delta$ sia autoaggiunto in $L^{2}\left(\boldsymbol{R}^{n}\right)$ è che abbia dominio nelle $f \in L^{2}\left(\boldsymbol{R}^{n}\right)$ tali che $\hat{f}(\lambda)|\lambda|^{2} \in L^{2}\left(\boldsymbol{R}^{n}\right)$, cioè tali che $\left(1+|\lambda|^{2}\right) \hat{f}(\lambda) \in L^{2}\left(\boldsymbol{R}^{n}\right)$, cioè abbia per dominio lo spazio di Sobolev $W^{2,2}\left(\boldsymbol{R}^{n}\right)$. 
È noto [11] che esiste un sistema o.n.c. in $L^{2}$ della sfera unitaria di $\boldsymbol{R}^{n}$ costituito da armoniche sferiche che si possono denotare $y_{k j}$, ove $k \in N$ è il grado del polinomio armonico da cui provengono e

$$
j=1, \ldots, N(k, n)=\frac{n+2 k-2}{n+k-2}\left(\begin{array}{c}
n+k-2 \\
k
\end{array}\right),
$$

che è il numero di armoniche sferiche lineramente indipendenti di grado $k$ in dimensione $n$. Volendo allora definire un sistema o.n.c. per $L^{2}\left(S_{t}, \omega_{t}\right)$, ove $\omega_{t}$, che è stata definita nel n. 2, è tale che $d \omega_{t}=t^{n / 2-1} / 2(2 \pi)^{n} d S_{1}$ (essendo $d S_{1}$ l'elemento di area sulla sfera unitaria) poniamo ovviamente $\left(^{1}\right) h_{k j}(t, \lambda)=2(2 \pi)^{n / 2} t^{\frac{1}{2}-n / 4} y_{k j}(\tilde{\lambda})$ con $\tilde{\lambda}=\lambda /|\lambda|$. Ora $(W f)_{k j}(t)=\left(f, \varphi_{k j}(t)\right)$ ove (n. 2$)$

$$
\varphi_{k j}(t)=\int_{|\lambda|^{2}=t} \exp [i(x, \lambda)] h_{k j}(t, \lambda) d \omega_{t}=\int_{|\lambda|^{2}=t} \exp [i(x, \lambda)] \sqrt{2}(2 \pi)^{n / 3} t^{\frac{1}{2}-n / 4} y_{k i}(\tilde{\lambda}) d \omega_{t}
$$

che è ben definito, perchè l'ultimo membro è l'integrale su un compatto di una funzione continua. Utilizzando ([17], pag. 31) l'eguaglianza:

$$
\exp [ \pm i(x, \lambda)]=(2 \pi)^{n / 2} \sum_{k j}( \pm i)^{k} y_{k j}(\tilde{\lambda}) y_{k j}(\tilde{x}) \frac{J_{n / 2+k-1}(|x||\lambda|)}{(|x||\lambda|)^{n / 2-1}}
$$

si ottiene

$$
\begin{aligned}
& \varphi_{k j}(t)=\sqrt{2}(2 \pi)^{n / 2} t^{\frac{1}{2}-n / 4} \int_{|\lambda|=1} \sum_{k^{\prime} j^{\prime}}(2 \pi)^{n / 2} i^{k^{\prime}} \frac{J_{n / 2+k-1}(|x| \sqrt{t})}{(|x| \sqrt{t})^{n / 2-1}} y_{k^{\prime} j^{\prime}}(\tilde{x}) y_{k^{\prime} j^{\prime}}(\tilde{\lambda}) y_{k j}(\tilde{\lambda}) \frac{t^{n / 2-1}}{2(2 \pi)^{n / 2}} d S_{1}(\tilde{\lambda}) \\
& =\frac{i^{k}}{\sqrt{2}}|x|^{1-n / 2} J_{n / 2+k-1}(|x| \sqrt{t}) y_{k j}(\tilde{x}) .
\end{aligned}
$$

Si osservi che è lecito scambiare i segni di serie e integrale in quanto la convergenza della serie a secondo membro di (2) è uniforme sui compatti e si ricordi che la successione delle funzioni $y_{k j}$ è un sistema o.n.

Le $\varphi_{k j}(\cdot, t) \in C^{\infty}\left(\boldsymbol{R}^{n}\right)$, poichè $|x|^{k} y_{k j}(x)$ è un polinomio e

$$
(|x| \sqrt{t})^{1-k-n / 2} J_{n / 2+\bar{k}-1}(|x| \sqrt{\imath})
$$

è una funzione trascendente intera in $t|x|^{2}$. Inoltre poichè $\left|J_{n / 2+k-1}(r)\right|=O\left(r^{-\frac{1}{2}}\right)([19]$, pag. 199), si ha che $\varphi_{k j} \in L^{p}\left(\boldsymbol{R}^{n}\right)$ per $p>2 n /(n-1)$. (Cfr. Il Teorema 2 di [17], pag. 192).

Rileviamo anche che $\varphi_{k j} \notin L^{2}\left(\boldsymbol{R}^{n}\right)$; pertanto sono soltanto autofunzioni generalizzate di $(-4)$, in quanto operatore su $W^{2,2}\left(\boldsymbol{R}^{n}\right)$. Serviamoei ora della eguaglianza:

(1) La scelta si è fatta per normalizzare $h_{k j}$ su $L^{2}\left(S^{a}\right)$; infatti

$$
\left\|h_{k j}\right\|_{L^{2}\left(S_{i}\right)}=\int_{S_{i}}\left|\sqrt{2}(2 \pi)^{n / 2} t^{\frac{1}{2}-n / 4} y_{k j}(\tilde{\lambda})\right|^{2} d \omega_{t}=\int_{|\alpha|=1}\left|y_{k j}\right|^{2} d S_{1}=1 .
$$


$f(x)=\left(W^{*} W f\right)(x)$ già provata nel n. 2 per ottenere la sintesi spettrale di $f$ e dello stesso n. 2, ricordiamo le formule

$$
(W f)(t)=\sum_{k j}\left[\int_{S t}\left(\lim _{r \rightarrow \infty} \int_{S(0, r)} f(x) \exp [-i(x, \lambda)] d x\right) \overline{h_{k j}(t, \lambda)} d \omega_{t}\right] h_{k j}(t, \lambda)
$$

per ogni $f \in H=L^{2}\left(\boldsymbol{R}^{n},||\right)$ il limite essendo fatto in $K=L^{2}\left(\boldsymbol{R}^{n}, \mid / /(2 \pi)^{n}\right)$ la serie convergendo in $L=\int_{N} H_{t} d t$.

$$
\left(W^{*} g\right)(x)=\lim _{s \rightarrow \infty} \int_{S(0,8)} \exp [i(x, \lambda)] \sum_{k j}\left[\left(\int_{s_{|\lambda|^{2}}} g\left(|\lambda|^{2}, \lambda\right) \overline{h_{k j}\left(|\lambda|^{2}, \lambda\right)} d \omega_{t}\right) h_{k j}\left(|\lambda|^{2}, \lambda\right)\right] \frac{d \lambda}{(2 \pi)^{n}}
$$

per ogni $g \in L$ ove il limite è fatto in $H$ e la serie converge in $K$. Cominciamo col provare che per ogni $f \in L^{2}\left(\boldsymbol{R}^{n}\right)$ è lecito scrivere

$$
(W f)(t)=\sum_{k, j}\left[\lim _{r \rightarrow \infty} \int_{S(0, r)} f(x) \overline{\varphi_{k j}(t, x)} d x\right] h_{k j}(t, \lambda)
$$

ove il limite è preso in $L^{2}(N)$ e la serie converge in $L$ : infatti posto $\hat{f}_{r}(\lambda)=$ $=\int_{S(0, r)} f(x) \exp [-i(x, \lambda)] d x$ si ha

$$
(W f)(t)=\sum_{k j}\left[\int_{S_{t}} \lim _{r \rightarrow \infty} \hat{f}_{r}(\lambda) \overline{h_{k j}(t, \lambda)} d \omega_{t}\right] h_{k j}(t, \lambda)=\sum_{k_{j} j}\left[\lim _{r \rightarrow \infty} \int_{S_{t}} \hat{f}_{r}(\lambda) \overline{h_{k j}(t, \lambda)} d \omega_{t}\right] h_{k j}(t, \lambda)
$$

poichè

$$
\begin{aligned}
& \left\|\int_{S_{t}} \lim _{r \rightarrow \infty} \hat{f}_{r}(\lambda) \overline{h_{k j}(t, \lambda)} d \omega_{t}-\int_{S_{t}} \hat{f}_{r}(\lambda) \overline{h_{k j}(t, \lambda)} d \omega_{t}\right\|_{L^{2}(N)}^{2}= \\
& =\iint_{N} \mid \int_{S_{t}}\left[\lim _{r \rightarrow \infty} \hat{f}_{r}(\lambda)-\hat{f}_{r}(\lambda)\right] \overline{h_{k j}(t, \lambda)} d \omega_{t}{ }^{2} d t \leqslant \int\left\|_{N}\right\| \lim _{r \rightarrow \infty} \hat{f}_{r}(\lambda)-\hat{f}_{r}(\lambda) \|_{L^{2}\left(S_{t}\right)}^{2} d t \\
& =\left\|\lim _{r \rightarrow \infty} \hat{f}_{r}(\lambda)-\hat{f}_{r}(\lambda)\right\|_{R}^{2}
\end{aligned}
$$

infinitesimo per $r \rightarrow \infty$. Allora utilizzando il teorema di Fubini

$$
\begin{aligned}
(W f)(t)=\sum_{k j}\left[\lim _{r \rightarrow \infty} \int_{S(0, r)} f(x)\left(\int_{S_{t}} \exp [-i(x, \lambda)] \overline{h_{k j}(t, \lambda)} d \omega_{t}\right) d x\right] h_{k j}(t, \lambda)= \\
=\sum_{k j}\left[\lim _{r \rightarrow \infty} \int_{S(0, r)} f(x) \overline{\varphi_{k j}(t, x)} d x\right] h_{k j}(t, \lambda) .
\end{aligned}
$$

Veniamo allora alla sintesi spettrale, vogliamo cioè provare che, indicato con

$$
(W f)_{k j}(t)=\lim _{r \rightarrow \infty} \int_{S(0, r)} f(x) \overline{\varphi_{k j}(t, x)} d x,
$$


si ha

$$
f(x)=\lim _{s \rightarrow \infty} \int_{0}^{s} \sum_{k j}(W f)_{k j}(t) \varphi_{k j}(t, x) d t
$$

ove il limite è fatto in $H$ e la serie converge in $L^{1}([0, s])$. Infatti

$$
\begin{aligned}
& f(x)=\left(W^{*} W f\right)(x)=\lim _{s \rightarrow \infty} \int_{s(0, s)} \exp [i(x, \lambda)] \\
& \cdot \sum_{k i}\left[\int_{\left.s_{i} \lambda\right|^{2}}\left(\sum_{k^{\prime} j^{\prime}}(W f)_{k^{\prime} j^{\prime}}\left(|\lambda|^{2}\right) h_{k^{\prime} j^{\prime}}\left(|\lambda|^{2}, \lambda\right) \overline{h_{k j}\left(|\lambda|^{2}, \lambda\right)}\right) d \omega_{t}\right] h_{k j}\left(|\lambda|^{2}, \lambda\right) \frac{d \lambda}{(2 \pi)^{3 /}}= \\
& =\lim _{s \rightarrow \infty} \int_{S(0, s)} \exp [i(x, \lambda)] \sum_{k, j}(W f)_{k j} \mid\left(|\lambda|^{2}\right) h_{k j}\left(|\lambda|^{2}, \lambda\right) \frac{d \lambda}{(2 \pi)^{n}}= \\
& =\lim _{s \rightarrow \infty} \int\left[\int_{0}^{s} \sum_{S_{t}}(W f)_{k j}(t) h_{k j}(t, \lambda) \exp [i(x, \lambda)] d \omega_{t}\right] d t= \\
& =\lim _{s \rightarrow \infty} \int_{0}^{s} \sum_{k j}(W f)_{k j}(t)\left[\int_{s_{t}} \exp [i(x, \lambda)] h_{k j}(t, \lambda) d \omega_{t}\right] d t
\end{aligned}
$$

ove la serie converge in $L^{1}([0, s])$, infatti

$$
\begin{aligned}
& \| \int_{S_{t}} \sum_{k j}(W f)_{k j}(t) h_{k j}(t, \lambda) \exp [i(x, \lambda)] d \omega_{t}- \\
& -\sum_{\substack{j=1 \\
k, \ldots \leqslant p}} \int_{S_{t}}(W f)_{k j}(t, \lambda) h_{k j}(t, \lambda) \exp [i(x, \lambda)] d \omega_{t} \|_{L^{1}([0, s])}= \\
& =\int_{0}^{s}\left|\int_{S_{t}} \sum_{j=1} \sum_{\substack{\ldots, N(k, n) \\
k>p}}(W f)_{k j}(t) h_{k j}(t, \lambda) \exp [i(x, \lambda)] d \omega_{t}\right| d t \leqslant \\
& \leqslant\left\{\int_{0}^{8} \int_{S_{t}}\left|\sum_{j=1, \ldots(k, n)}(W f)_{k j}(t) h_{k j}(t, \lambda)\right|^{2} d \omega_{t} d t\right\}^{\frac{1}{2}}|S(0, s)|^{\frac{1}{2}}
\end{aligned}
$$

infinitesima per $p \rightarrow \infty$. Allora

$$
f(x)=\lim _{s \rightarrow \infty} \int_{0}^{s} \sum_{k j}(W f)_{k j}(t) \varphi_{k j}(t, x) d t .
$$

Tale integrale coincide esattamente con l'espressione di $P(s) f$ data nel teorema 4 di [17] pag. 200.

Osservazione. - Per il laplaciano iterato $(-\Delta)^{m}$ si procede analogamente, solo si considerano le $S_{t}$ definite dall'equazione $|\lambda|=t^{1 / 2 m}$. Allora si hanno le seguenti 
modifiche

$$
h_{k j}(t, \lambda)=\sqrt{2 m}(2 \pi)^{n / 2} t^{\frac{4}{3}-n / 4 m} y_{k j}(\tilde{\lambda})
$$

per cui

$$
\varphi_{k j}(t, x)=\frac{i^{k}}{\sqrt{2 m}} t^{(1-m) / 2 m}|x|^{1-n / 2} J_{n / 2+k-1}\left(|x| t^{1 / 2 m}\right) y_{k j}(\tilde{x})
$$

\section{4. - Laplaciano nel semispazio e nello strato.}

Consideriamo il Laplaciano avente come dominio $\left\{f \in W^{2,2}(\Omega),\left(\partial f / \partial x_{n}\right),\left(x_{1}, \ldots\right.\right.$, $\left.x_{n-1}, 0\right)=0$ nel senso delle tracee $\}$ ove $\Omega=\left\{\left(x_{1}, \ldots, x_{n}\right) \in \boldsymbol{R}^{n}, x_{n}>0\right\}$. Definiamo l'operatore $U: L^{2}(\Omega) \rightarrow L^{2}\left(\boldsymbol{R}^{n}\right)$ mediante $(U f)(\lambda)=\int_{\Omega} f(x) \varphi(x, \lambda) d x$, se $f \in C_{0}^{\infty}(\Omega)$ ove $\varphi(x, \lambda)=\exp \left[-i \sum_{j=1}^{n-1} x_{j} \lambda_{j}\right] \cos \lambda_{n} x_{n}$ e prolungato su $L^{2}(\Omega)$ per continuità. Poichè combinazioni lineari delle funzioni $f(x)=g\left(x_{1}, \ldots, x_{n-1}\right) h\left(x_{n}\right),\left(g \in C_{0}^{\infty}\left(\boldsymbol{R}^{n-1}\right), h \in C_{0}^{\infty}\left(\boldsymbol{R}_{+}\right)\right)$sono dense in $L^{2}(\Omega)$, si ha che $U$ è unitario, grazie anche al teorema di Parseval ed alla proprietà della trasformazione cos ([5], eap. XIII). Inoltre $U$ diagonalizza l'operatore con autovalore $|\lambda|^{2}$; infatti indicato con $\Delta_{n-1}=\sum_{j=1}^{n-1} \partial^{2} / \partial x_{j}^{2}$ si ha $\Delta=\Delta_{n-1}+\partial^{2} / \partial x_{n}^{2}$ : allora se $f \in C_{0}^{\infty}(\bar{\Omega})$ (che è denso nel dominio dell'operatore considerato cfr. [3], lemma 10 , pag. 50 con qualche breve modifica) risulta

$$
\begin{aligned}
& U(-\Delta) f=\int \exp \left[-i \sum_{j=1}^{n-1} x_{j} \lambda_{j}\right] \cos \lambda_{n} x_{n}(-\Delta) f(x) d x= \\
& \quad=\int\left[\left(-\Delta_{n-1}\right) \exp \left[-i \sum_{j=1}^{n-1} x_{j} \lambda_{j}\right] \cos \lambda_{n} x_{n}-\exp \left[-i \sum_{j=1}^{n-1} x_{j} \lambda_{j}\right]\left(\frac{\partial^{2}}{\partial x_{n}^{2}} \cos \lambda_{n} x_{n}\right)\right] f(x) d x= \\
& \quad=\left(\lambda_{1}^{2}+\ldots+\lambda_{n-1}^{2}+\lambda_{n}^{2}\right) \int \exp \left[-i \sum_{j=1}^{n-1} x_{j} \lambda_{j}\right] \cos \lambda_{n} x_{n} f(x) d x=|\lambda|^{2}(U f)(\lambda) .
\end{aligned}
$$

Basta allora applicare la $V$ del n. 3 per ottenere la diagonalizzazione canonica e in modo analogo la sintesi spettrale.

Prendiamo in considerazione il laplaciano avente come dominio $\left\{f \in W^{2,2}(\Omega)\right.$ : $: f\left(x_{1}, \ldots, x_{n-1}, 0\right)=f\left(x_{1}, \ldots, x_{n-1}, l\right)=0$ nel senso delle tracee $\}$ ove $\Omega=\left\{\left(x_{1}, \ldots, x_{n}\right) \in\right.$ $\left.\in \boldsymbol{R}^{n} 0<x_{n}<l\right\}$. Sia $M=\{m \pi / l, m \in \boldsymbol{N}\}$ e definiamo l'operatore

$$
U: L^{2}(\Omega) \rightarrow L^{2}\left(\boldsymbol{R}^{n-1} \times M\right)
$$

tale che

$$
(U f)\left(\lambda_{1}, \ldots, \lambda_{n-1}, \frac{m \pi}{l}\right)=\int_{\Omega} f(x) \varphi\left(x, \lambda_{1}, \ldots, \lambda_{n-1}, \frac{m \pi}{l}\right) d x
$$


ove

$$
\varphi\left(x, \lambda_{1}, \ldots, \lambda_{n-1}, \frac{m \pi}{l}\right)=\sqrt{\frac{2}{\pi}} \exp \left[-i \sum_{j=1}^{n-1} x_{j} \lambda_{j}\right] \text { sen } \frac{m \pi}{l} x_{n}, \quad \text { se } f \in C_{0}^{\infty}(\Omega)
$$

e prolungato su $L^{2}(\Omega)$ per continuità.

Come precedentemente $U$ è unitario; proviamo che diagonalizza l'operatore con autovalori $\lambda_{1}^{2}+\ldots+\lambda_{n-1}^{2}+\left(m^{2} \tau^{2}\right) / l^{2}$.

Sia $f \in C_{0}^{\infty}(\bar{\Omega})$, che è denso nl dominio dell'operatore considerato, [3] lemma 10 pag. 50 ,

$$
\begin{aligned}
& (U(-\Delta) f)(\lambda)=\int \sqrt{\frac{2}{\pi}} \exp \left[-i \sum_{j=1}^{n-1} x_{j} \lambda_{j}\right]\left(\operatorname{sen} \frac{m \pi}{l} x_{n}\right)(-\Delta) f(x) d x= \\
& =\int\left[\left(-\Delta_{n-1}\right)\left(\sqrt{\frac{2}{\pi}} \exp \left[-i \sum_{j=1}^{n-1} x_{j} \lambda_{j}\right] \operatorname{sen} \frac{m \pi}{l} x_{n}\right)+\exp \left[-i \sum_{j=1}^{n-1} x_{j} \lambda_{j}\right]\left(\frac{\partial^{2}}{\partial x_{n}^{2}} \operatorname{sen} \frac{m \pi}{l} x_{n}\right)\right] f(x) d x \\
& =\left(\lambda_{1}^{2}+\ldots+\lambda_{n-1}^{2}+\frac{m^{2} \pi^{2}}{l^{2}}\right) \int \sqrt{\frac{5}{\pi}} \exp \left[-i \sum_{j=1}^{n-1} x_{j} \lambda_{j}\right]\left(\operatorname{sen} \frac{m \pi}{l} x_{n}\right) f(x) d x= \\
& =\left(\lambda_{1}^{2}+\ldots+\lambda_{n-1}^{2}+\frac{m^{2} \pi^{2}}{l^{2}}\right)(U f)(\lambda) .
\end{aligned}
$$

Per ottenere la diagonalizzazione canonica basta costruire un operatore $V$ come nel n. 2, osservando però che essendo ora $p\left(\boldsymbol{R}^{n-1} \times M\right)=\left[(\pi / l)^{2}, \infty\right)$ lintegrale diretto è ora:

$$
\int_{(\pi / h)^{2}}^{\infty} L^{2}\left(\boldsymbol{S}_{t}\right) d t, \quad \text { ove } \quad S_{t}=\left\{\lambda \in \boldsymbol{R}^{n-1} \times M: p(\lambda)=t\right\}
$$

Analogamente a quanto si è visto nel n. 3 si ottiene la sintesi spettrale.

\section{5. - Laplaciano all'esterno di $S_{a^{\mathrm{n}}}$.}

Studiamo ora il laplaciano avente dominio $\left\{f \in W^{2 \cdot 2}(\Omega): f=0\right.$ su $S_{a^{2}}$ nel senso delle tracce $\}$ essendo $\Omega=\boldsymbol{R}^{n} \backslash \overline{S(0, a)}$. Occorre ora modificare la definizione della $U$ del n. 2 ed anzi avremo bisogno di introdurla per mezzo di alcune funzioni supplementari.

Consideriamo $U_{a}: L^{2}(\Omega) \rightarrow L^{2}\left(\boldsymbol{R}^{n}\right)$ definito ponendo per $f \in C_{0}^{\infty}(\Omega)$

$$
\left(U_{a} f\right)(\lambda)=\int_{\Omega} f(x) \overline{F_{a}(x, \lambda)} d x
$$

ove

$$
\begin{gathered}
F_{a}(x, \lambda)=w_{a}(x, \lambda,|\lambda|), \quad w_{a}(x, \lambda, l)=\exp [i(x, \lambda)]+v_{a}(x, \lambda, l) \\
v_{a}(x, \lambda, l)=-\sum_{k j}(2 \pi)^{n / 2} i^{k} \frac{y_{k j}(\tilde{x}) y_{k j}(\tilde{\lambda})}{(|x| \mid \lambda)^{n / 2-1}} J_{n / 2+k-1}(a|\lambda|) \frac{H_{n / 2+k-1}(|x| l)}{H_{n / 2+k-1}(a l)}
\end{gathered}
$$


(con $J_{y}$ e $H_{p}$ si sono indicate le funzioni di Bessel di prima e terza specie [19] e $v_{a} \grave{\mathrm{e}}$ considerata per $x \in \bar{\Omega}, \lambda \in \boldsymbol{R}^{n}, l \in \boldsymbol{C}$ tale che $\left.l^{2}=\mu+i \varepsilon, \mu \geqslant 0,0 \leqslant \varepsilon \leqslant \varepsilon_{0}, \varepsilon_{0}>0, \operatorname{Im} l \geqslant 0\right)$. Ci sarà utile mettere in evidenza alcuni fatti relativi a $v_{a}$ : innanzitutto che $v_{a}$ è ben definita: proviamo pereiò prechè la serie che la definisce è convergente.

Allo scopo cominciamo col notare che esiste una costante $M$ (indipendente da $v$ e da $x \in \Omega)$ tale che sia $\left|H_{v}(|x| l) / H_{\nu}(a l)\right| \leqslant M$ per ogni

$$
l \in T=\{l \in C: \operatorname{Re}(l) \geq 0, \operatorname{Im}(l) \geq 0\} .
$$

Ciò può essere dedotto tenendo presente $([19]$, pag. 78,511$)$ che la funzione $p(l)=$ $=H_{v}(|x| l) / H_{v}(a l)$ è olomorfa in $\dot{T}$, allora il suo massimo modulo in domini del tipo $T \cap\{l:|l|<R\}$ è assunto sulla frontiera, e quindi l'uniforme limitetazza in $T$ di $\varphi(l)$ rispetto ad $x$ e $V$ segue dalla stima:

$$
H_{v}(z)=\left(\frac{2}{\pi z}\right)^{\frac{1}{2}} \exp \left[i\left(z-\frac{1}{2} v \pi-\frac{1}{4} \pi\right)\right]\left(1+o\left(\frac{1}{z}\right)\right)
$$

([19], pag. 198) e dal fatto che le funzioni $t \mapsto H_{v}(|x| t)$ e $t \mapsto H_{v}(|x| i t)$ sono decrescenti in $\boldsymbol{R}_{+}$([19], pag, 487-185).

Osserviamo ora che è:

$$
\sum_{k j}(2 \pi)^{n / 2} i^{k} \frac{y_{k j}(\tilde{x}) y_{k j}(\tilde{\lambda})}{(|x||\lambda|)^{n / 2-1}} J_{n / 2+k-1}(a|\lambda|)=\left(\frac{a}{|x|}\right)^{n / 2-1} \exp [i(\lambda, a \tilde{x})]
$$

e proviamo che tale serie converge assolutamente in $Q \times \boldsymbol{R}^{n}$. Da [19], pag. 49 si ha

$$
\left|J_{\nu}(t)\right| \leqslant \frac{\left|\frac{1}{2} t\right|^{\nu}}{\Gamma(\nu+1)} \exp [|\operatorname{Im} t|]
$$

da $[15]$, pag. 32

$$
\frac{2 \pi^{n / 2}}{\Gamma(n / 2-1)} \sum_{j=1}^{N(k, n)} y_{k j}(\tilde{x}) y_{k j}(\tilde{\lambda})=\left(\frac{n}{2}+k-1\right) O_{k}^{n / 2-1}\left(\cos \frac{(x, \lambda)}{|x||\lambda|}\right)
$$

e da [9], pag. 222

$$
\left|C_{m}^{k} \cos \varphi\right| \leqslant 2^{2 m+k}
$$

mettendo assieme queste disuguaglianze si ottiene:

$$
\begin{aligned}
\left|\frac{2 \pi^{n / 2}}{\Gamma(n / 2-1)} i^{i k} \sum_{j=1}^{N\left(k_{k} n\right)} \frac{y_{k j}(\tilde{x}) y_{k j}(\tilde{\lambda})}{(|x||\lambda|)^{n / 2-1}} J_{n / a+k-1}(a|\lambda|)\right| \leqslant & \\
& \leqslant \frac{2^{n / 2+k-1}}{(|\alpha||\lambda|)^{n / 2-1}} \frac{(|\lambda| a)^{k}}{\Gamma(n / 2+k)} \leqslant \frac{2^{n / 2+k-1}}{\Gamma(n / 2+k)}(|\lambda| a)^{k} .
\end{aligned}
$$


Ciò prova la convergenza assoluta in $\Omega \times \boldsymbol{R}^{n} \times T$ della serie che definisce $v_{a}(x, \lambda, l)$ e la continuità della funzione stessa.

Inoltre per (2) $v_{a}(x, \lambda, l)=-\exp [i(x, \lambda)]$ su $S_{a^{2}}$, in modo che $w_{a}(x, \lambda, l)=0$ su $S_{a^{3}}$. Infine $v_{a}(x, \lambda, l) \in L^{p}(\Omega)$ per $p>2 n /(n-1)$, come subito si verifica, rifacendo uso della (3) e del principio di massimo applicato alla funzione

$$
\psi(l)=\frac{|x|^{\frac{1}{2}} H_{n / 2+k-1}(|x| l)}{a^{\frac{1}{2}} H_{n / 2+k-1}(a l)}
$$

su domini del tipo sopra considerato, quando si tenga presente le proprietà di decrescenza delle funzioni $t \mapsto t^{\frac{1}{2}} H_{v}(|x| t)$ e $t \mapsto t^{\frac{1}{2}} H_{v}(|x| i t)$ in $\boldsymbol{R}_{+}$([19], pag. 446 e 206).

Osserviamo che $v_{a}(\cdot, \lambda, l)$ è autosoluzione (generalizzata) di $-\Delta$ con autovalore $l^{2}$. A questo scopo verifichiamo con un ben noto classico ragionamento (che riportiamo per comodità del lettore) che lo è la funzione $g_{l}(|x|) h(x)=y_{k j}(\tilde{x})\left(H_{n / 2+k-1}(|x| l) /|x|^{n / 2-1}\right)$ ove $g_{l}(|x|)=|x|^{-(n / 2+k-1)} H_{n / 2+k-1}(|x| l)$ e $h(x)=|x|^{k} y_{k j}(\tilde{x})$. Infatti

$$
\Delta\left(g_{l}(|x|) h(x)=g_{l}(|x|) \Delta h(x)+2 \frac{g_{l}^{\prime}(|x|)}{|x|} \sum_{j=1}^{n} x_{j} \frac{\partial h}{\partial x_{j}}(x)+h(x)\left(g_{l}^{\prime \prime}(|x|)+\frac{n-1}{|x|} g_{l}^{\prime}(|x|)\right) .\right.
$$
Ma $\Delta h(x)=0$ perchè $h(x)$ è polinomio armonico; $\sum_{j=1}^{n} x_{j}\left(\partial h / \partial x_{j}\right)(x)=k h(x)$, essendo
$h(x)$ polinomio omogeneo di grado $k$.

Si perviene dunque alle seguenti identità:

$$
\begin{aligned}
& \left(\Delta+l^{2}\right)\left(y_{k_{j}}(x) \frac{H_{n / 2+k-1}(|x| l)}{|x|^{n / 2-1}}=\Delta\left(g_{l}(|x|) h(x)\right)+l^{2}\left(g_{l}(|x|) h(x)\right)\right)= \\
& =h(x)\left[g_{l}^{\prime \prime}(|x|)+\frac{n+2 k-1}{|x|} g_{b}^{\prime}(|x|)+l^{2} g_{l}(|x|)\right]= \\
& =h(x)\left[\left(\frac{n}{2}+k-1\right)\left(\frac{n}{2}+k\right)|x|^{-(n / 2+k-1)} H_{n / 2+k-1}(|x| l)+\right. \\
& +2\left(\frac{n}{2}+k-1\right) l|x|^{-(n / 2+k)} H_{n / 2+k-1}^{\prime}(|x| l)+l^{2}|x|^{-(n / 2+k-1)} H_{n / 2+k-1}^{\prime \prime}(|x| l)+ \\
& -(n+2 k-1)\left(\frac{n}{2}+k-1\right)|x|^{-(n / 2+k-1)} H_{n / 2+k-1}(|x| l)+(n+2 k-1) l|x|^{-(n / 2+k)} H_{n / 2+k-1}^{\prime}(|x| l)+ \\
& \left.+l^{2}|x|^{\mid n / 2+k-1} H_{n / 2+k-1}(|x| l)\right]=h(x) l^{2}|x|^{-(n / 2+k-1)} . \\
& \quad\left[H_{n / 2+k-1}^{\prime \prime}(|x| l)+\frac{H_{n / 2+k-1}(x l)}{|x| l}+\left(1-\left(\frac{n}{2}+k-1\right)^{2} \frac{1}{l^{2}|x|^{2}}\right) H_{n / 2+k-1}(|x| l)\right]
\end{aligned}
$$

che è appunto nullo. 
Vogliamo ora provare che per ogni $\lambda \in \boldsymbol{R}^{n}$ e $l \in \boldsymbol{C}$ valgono le seguenti relazioni $\left|\frac{\partial v_{a}}{\partial|x|}-i\right| \lambda\left|v_{a}\right|=o\left(\frac{1}{|x|^{\frac{1}{(2 n-1)}}}\right) \quad$ e $\quad v_{a}(x, \lambda, l)=0\left(\frac{\exp [-(\operatorname{Im} l)|x|]}{|x|^{\mid \frac{1(n-1)}{(n)}}}\right) \quad$ per $|x| \rightarrow \infty$,

la prima delle quali è nota sotto il nome di condizione di radiazione di Sommerfeld.

Per far questo occorre derivare sotto il segno di serie e dunque abbiamo bisogno della convergenza uniforme della serie delle derivate, questa si prova tenendo conto che: $H_{v}^{\prime}(z)=H_{v-1}(z)-(v / z) H_{v}(z)\left([19]\right.$, pag. 74), del fatto che $H_{v}(|x||\lambda|)$ è funzione crescente di $\nu$ ([19], pag. 444) e tenendo conto della stima precedente (3).

Allora operando sugli addenti, poichè $H_{p-1}(z)-H_{v+1}(z)=2 H_{v}^{\prime}(z)([19]$, pag. 74) e

$$
\begin{array}{r}
H_{v}(z)=\left(\frac{2}{\pi z}\right)^{\frac{1}{2}} \exp \left[i\left(z-\frac{1}{2} v \pi-\frac{\pi}{4}\right)\right]\left(1-\frac{4 v^{2}-1}{8 i z}+0\left(\frac{1}{|z|^{2}}\right)\right) \\
([19], \text { pag. 198) se }|z| \rightarrow \infty
\end{array}
$$

si ha che:

$$
\lim _{|x| \rightarrow \infty}|x|^{\frac{1}{2}(n-1)}\left(\frac{\partial}{\partial x} \frac{H_{n / 2+k-1}(|x||\lambda|)}{|x|^{\mid n / 2-1}}-i|\lambda| \frac{H_{n / 2+k-1}(|x||\lambda|)}{|x|^{n / 2-1}}\right)=0
$$

La condizione di Sommerfeld è allora verificata, essendo $1 /\left|H_{y}(a|\lambda|)\right|$ limitato al variare di $v$ ([19], pag. 511, 444), il che fa sì che per la (3)

$$
\sum_{k j}(2 \pi)^{n / 2} i^{k} \frac{y_{k j}(\tilde{x}) y_{k j}(\tilde{\lambda})}{(|x||\lambda|)^{n / 2-1}} \frac{J_{n / 2+k-1}(a|\lambda|)}{H_{n / 2+k-1}(a|\lambda|)}
$$

converga assolutamente. Il secondo comportamento enunciato per $v_{a}(x, \lambda, l)$ all' $\infty$ segue anch'esso facilmente dalle precedenti stime.

Serviamoci delle proprietà precedenti per provare che $U_{\alpha}$ è unitaria: la surgettività segue da [8] per $n=3$ (ma il ragionamento può essere esteso al caso generale [2]) oppure da [15]. Proviamo che $U_{a}$ è isometria.

Ora sia $H(x, \cdot, l)\left(\in L^{i} \cap L^{2}\right)$ la funzione di Green del problema

$$
\begin{cases}\left(-\Delta-l^{2}\right) u=f \\ u=0 \quad \text { su } \partial \Omega\end{cases}
$$

(per l'esistenza v. [14] pag. 655) e sia $u(x, \lambda, l)=\breve{H}(x, \cdot, l)$, ove $H(x, y, l)$ si intende prolungata o fuori di $\Omega$ e $\checkmark$ è l'antitrasformata di Fourier. Vogliamo ora provare chè è:

$$
(2 \pi)^{n / 2}\left(|\lambda|^{2}-l^{2}\right) u(x, \lambda, l)=\exp [i(x, \lambda)]+v_{a}(x, \lambda, l)
$$

Infatti $\left(-\Delta-l^{2}\right)\left[\exp [i(y, \lambda)]+v_{o}(y, \lambda, l)\right]=\left(|\lambda|^{2}-l^{2}\right) \exp [i(y, \lambda)]$, moltiplicando 
ambo i membri per $H(x, y, l)$ e integrando in $d y$ si ottiene

$\int H(x, y, l)\left(-\Delta-l^{2}\right)\left[\exp [i(y, \lambda)]+v_{a}(y, \lambda, l)\right] d y=\left(|\lambda|^{2}-l^{2}\right) \int H(x, y, l) \exp [i(y, \lambda)] d y$

da cui la relazione voluta, poichè la funzione di Green è il nucleo dell'inverso di $-\Delta-l^{2}$.

Dal teorema di Parseval, otteniamo:

$$
\begin{aligned}
& \int H(z, x, l) \overline{H(z, y, l)} d z=\frac{1}{(2 \pi)^{n}\left(l^{2}-\bar{l}^{2}\right)} \cdot \\
& \cdot \int\left[\frac{1}{|\lambda|^{2}-l^{2}}+-\frac{1}{|\lambda|^{2}-\bar{l}^{2}}\right] w_{a}(x, \lambda, l) \overline{w_{a}(x, \lambda, l)} d \lambda .
\end{aligned}
$$

Moltiplichiamo ora i due membri per $\overline{f(x)} f(y)$, integriamo su $\Omega \times \Omega$ in $d x d y$ e moltiplichiamo ancora per $l^{2}-\bar{l}^{2}$. Dal primo membro si ottiene $\left(l^{2}-\bar{l}^{2}\right)\left(R_{\bar{l}^{2}} f, R_{l^{2}} f\right)=$ $=\left(l^{2}-\bar{l}^{2}\right)\left(R_{\bar{l}^{2}} R_{i^{2}} f, f\right)=\left(\left(R_{l^{3}}-R_{\bar{l}^{2}}\right) f, f\right)$ avendo utilizzato la prima equazione risolvente ([7], pag. 16). Ora si definisce

$$
\Phi_{a}(\lambda, l)=(2 \pi)^{-n / 2} \int_{\Omega} \overline{w_{a}(x, \lambda, l)} f(x) d x, \quad f \in C_{0}^{\infty}(\Omega) .
$$

Sia $l^{2}=\mu+i \varepsilon:$ per $\mu \in[\alpha, \beta], 0 \leqslant \varepsilon \leqslant \varepsilon_{0}$ vale la diseguaglianza:

$$
\int \frac{1}{\left(1+|\lambda|^{2}\right)^{2}}\left|\Phi_{a}(x, \lambda, l)\right|^{2} d \lambda \leqslant M
$$

ove $M$ non dipende da $l\left([15]\right.$, pag. 130) e $\Phi_{a}(\lambda, l) \mapsto \int f \cdot \bar{F}_{a} d x$ se $l \rightarrow|\lambda|$. Poichè l'integrando del secondo membro di (4):

$$
\frac{2 i \varepsilon}{\left(|\lambda|^{2}-\mu\right)^{2}+\varepsilon^{2}} w_{a}\left(x, \lambda, \sqrt{\mu+i \varepsilon)} \overline{w_{a}(x, \lambda, \sqrt{\mu+i \varepsilon})}\right.
$$

è integrabile in $\lambda$, si perviene alla relazione:

Per [14], pag. 183

$$
\left.\left(R_{\mu_{+} \varepsilon \varepsilon}-R_{\mu_{-i \varepsilon}}\right) f, f\right)=\int \frac{2 i \varepsilon}{\left(|\lambda|^{2}-\mu\right)^{2}+\varepsilon^{2}}\left|\Phi_{a}(\lambda, \sqrt{\mu+i \varepsilon})\right|^{2} d \lambda
$$

$$
\frac{1}{2}\left[\left(\left(E_{\beta}+E_{\beta_{-0}}\right) f, f\right)-\left(\left(E_{\alpha}+E_{\alpha_{-0}}\right) f, f\right)\right]=\frac{1}{2 \pi i} \lim _{\varepsilon \rightarrow 0} \int_{\alpha}^{\beta}\left(\left(R_{\mu_{+i \varepsilon}}-R_{\mu_{-i \varepsilon}}\right) f, f\right) d \mu .
$$

Utilizzando allora il teorema di Fubini si ottiene:

$$
\frac{1}{2}\left(\left(E_{\beta}+E_{\beta_{-0}}\right) f, f\right)-\left(\left(E_{\alpha}+E_{\alpha-0}\right) f, f\right)=\frac{1}{2} \lim _{\varepsilon \rightarrow 0} \int d \lambda \int_{\alpha}^{\beta} \frac{\varepsilon}{\left(|\lambda|^{2}-\mu\right)^{2}+\varepsilon^{2}}\left|\Phi_{a}(\lambda, \sqrt{\mu+i \varepsilon})\right|^{2} d \mu .
$$


È lecito portare il limite sotto il primo segno di integrale [15], pag. 129. Tenuto conto di [18], pag. 31 si ottiene:

$$
\frac{1}{2}\left[\left(\left(E_{\beta}+E_{\beta-0}\right) f, f\right)-\left(\left(E_{\alpha}+E_{\alpha-0}\right) f, f\right)\right]=\int_{\sqrt{x} \leqslant|\lambda| \leqslant \sqrt{\beta}}\left|\int f \cdot \vec{F}_{a} d x\right|^{2} d \lambda
$$

Se ora si fa tendere $\alpha$ a $\left.\beta\left(E_{\beta}-E_{\beta-0}\right) f, f\right)=0 \Rightarrow E_{\beta}=E_{\beta-0}$, cioè non esistono autosoluzioni.

Allora $\left(\left(E_{\beta}-E_{\alpha}\right) f, f\right)=\int_{\sqrt{ } \times \mathbb{\alpha} \leqslant|\lambda| \leqslant \sqrt{\beta}}\left|\int f \cdot \bar{F}_{a} d x\right|^{2} d \lambda$, e se si fa tendere $\alpha$ a $0, \beta$ ad $\infty$, si ottiene: $\|f\|_{2}^{2}=\int\left|\int f \cdot \bar{F}_{a} d x\right|^{2} d \lambda$ per ogni $f \in C_{0}^{\infty}(\Omega)$, quindi $U_{a}$ ̀̀ isometria.

Per ogni $f \in C_{0}^{\infty}(\bar{\Omega})$ (che è denso nel dominio dell'operatore considerato, [3] lemma 10, pag. 50) vale $\nabla_{a}(-\Delta) f=|\lambda|^{2} U_{a} f$, infatti

$$
\begin{aligned}
U_{a}(-\Delta) f(\lambda)=\int_{\Omega}(-\Delta) f(x) \overline{F_{a}(x, \lambda)} d x=\int_{\Omega} f(x) \overline{(-\Delta) F_{a}(x, \lambda)} d x= \\
=|\lambda|^{2} \int_{\Omega} f(x) \overline{F_{a}(x, \lambda)} d x=|\lambda|^{2} U_{a} f(\lambda)
\end{aligned}
$$

poichè

$$
\begin{aligned}
(-\Delta) F_{a}(x, \lambda)=(-\Delta)(\exp [i(x, \lambda)]+ & \left.v_{a}(x, \lambda,|\lambda|)\right)= \\
& =|\lambda|^{2}\left(\exp [i(x, \lambda)]+v_{a}(x, \lambda,|\lambda|)\right)=|\lambda|^{2} F_{a}(x, \lambda) .
\end{aligned}
$$

Basta allora applicare l'operatore $V$ considerato nel n. 2 per ottenere la diagonalizzazione canonica, ed un sistema di autofunzioni generalizzate.

\section{BIBLIOGRAFIA}

[1] JV. M. Berezanskir, Expansion in Eigenfunetions of Selfadjoint operator, American Mathematical Society, Providence, 1968.

[2] G. F. BotraRo, Alcuni risultati di analisi spettrale per operatori differenziali a coefficienti costanti su insiemi non limitati, Le Matematiche, 29 (1974), pp. $20-41$.

[3] F. E. Browder, On the spectral theory of elliptie differential operator, Math. Ann., 142 (1961), pp. 22-130.

[4] J. Drxmier, Les algèbres d'opérateurs dans l'espace Hilbertien, Gauthier-Villars, Paris, 1969.

[5] N. Dunford - J. T. SchwarTz, Linear operators, vol. II, Interscience, 1963.

[6] L. GăRDING, Eigenfunction expansions, pp. 303-325 di: Bers - JoHN - SHECTER, Partial Differential Equations, Interscience, New York, 1964.

[7] E. Hinle - R. S. PHILIPs, Functional analysis and semigroups, Amer. Math. Soc. Colloq. Publ. 31, 1957.

[8] T. IKEBE. Orthogonality of the eigenfunctions for the exterior problem connected with $-\Delta$, Arch. Rat. Mech. Anal., 19 (1965), pp. 71-73.

[9] W. Magnus - F. Obermettinger - R. P. Sost, Formulas and theorems for the special functions of Mathematical Physics, III edition, Springer-Verlag, 1960. 
[10] K. MaURIN, Methods of Hilbert spaces, Polish Scientific Publ, Warszawa, 1967.

[11] C. MuLcer, Spherical harmonics, Lectures notes in mathematics, vol. 17, SpringerVeriag, Berlin, 1966.

[12] J. von NeUmaNN, On rings of operators. Reduction theory, Ann. Math., (2) 50 (1949), pp. $401-485$.

[13] F. Rresz - B. S7. NAGY, Legon d'analyse fonctionelle, Akademiai Kado, 1953.

[14] V. I. Smirnov, A course of higher Mathematics, vol. IV: Integral equations and partial differential equations, Pergamon Press, Londra, 1964.

[15] N. A. SHENK, Eigenfunction expansion and scattering theory for the wave equation in an exterior region, Arch. Rat. Mech. Anal., 21 (1966), pp. 120-150.

[16] M. H. Stone, Linear transformations in Hilbert spaces and their applications to analysis, Amer. Math. Soc. Colloq. Publ. 15, 1932.

[17] G. TALENT, Spectrum of the Laplace operator acting in $L^{p}\left(\boldsymbol{R}^{n}\right)$, Symp. Math. VII, Academic Press (1971), pp. 185-232.

[18] T. C. Trmohmarsh, Introduction to the theory of Fourier integrals, Clarendon Press, Oxford, 1937.

[19] G. N. Watson, A treatise of the theory of Bessel funetions, The Macmillan Company, New York, 1948. 